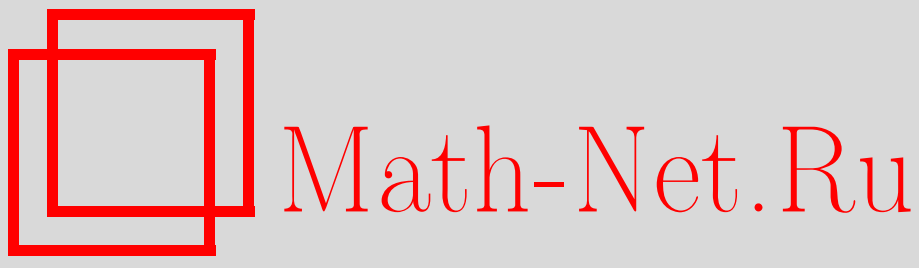

М. А. Чахкиев, О показателе сходимости особого интеграла многомерного аналога проблемы Терри, Изв. РАН. Сер. матем., 2003, том 67, выпуск 2, 211-224

DOI: https://doi.org/10.4213/im432

Использование Общероссийского математического портала Math-Net.Ru подразумевает, что вы прочитали и согласны с пользовательским соглашением http://www . mathnet.ru/rus/agreement

Параметры загрузки:

IP: 54.198 .67 .100

26 апреля 2023 г., $11: 22: 29$ 
УДК 517.5

\author{
М. А. Чахкиев
}

\title{
О показателе сходимости особого интеграла многомерного аналога проблемы Терри
}

\footnotetext{
Приведены оценки снизу и сверху показателя сходимости особого интеграла многомерного аналога проблемы Терри, связанной с асимптотикой числа решений систем диофантовых уравнений. В ряде случаев установлены точные значения показателя сходимости особого интеграла.

Библиографиия: 9 наименований.
}

1. Введение. Особый интеграл проблемы Терри, связанный с асимптотикой числа решений систем диофантовых уравнений (см. [1] и библиографию там же), имеет вид

$$
\theta=\int_{-\infty}^{+\infty} \cdots \int_{-\infty}^{+\infty}\left|\int_{0}^{1} \cdots \int_{0}^{1} \exp \left\{2 \pi i F\left(x_{1}, \ldots, x_{r}\right)\right\} d x_{1} \ldots d x_{r}\right|^{2 k} d \alpha
$$

где

$$
F\left(x_{1}, \ldots, x_{r}\right)=\sum_{t_{1}=0}^{n_{1}} \ldots \sum_{t_{r}=0}^{n_{r}} \alpha\left(t_{1}, \ldots, t_{r}\right) x_{1}^{t_{1}} \ldots x_{r}^{t_{r}}
$$

- многочлен с вещественными коэффициентами $\alpha=\alpha\left(t_{1}, \ldots, t_{r}\right), \alpha(0, \ldots, 0) \equiv 0$. Для неполной системы диофантовых уравнений часть коэффициентов $\alpha\left(t_{1}, \ldots, t_{r}\right)$ может быть тождественно равной нулю.

Число $\gamma$ называется показателем сходимости интеграла $\theta$, если он сходится при $2 k>\gamma+\varepsilon$ и расходится при $2 k<\gamma-\varepsilon$, где $\varepsilon-$ произвольное, сколь угодно малое положительное число. В одномерном случае, т.е. при $r=1$, в работах [1]-[6] проблема Хуа Ло-Гена [7] о показателе сходимости особого интеграла (1) решена полностью. В многомерном случае теми же авторами (см. [1]) получены оценки сверху показателя сходимости для многочлена (2):

$$
\gamma \leqslant n m,
$$

где $n=\max \left(n_{1}, \ldots, n_{r}\right), m=\left(n_{1}+1\right) \ldots\left(n_{r}+1\right)-1$, и для многочлена вида

$$
F\left(x_{1}, \ldots, x_{r}\right)=\sum_{\substack{t_{1}=0 \\ t_{1}+\cdots+t_{r} \leqslant n}}^{n} \ldots \sum_{\substack{t_{r}=0 \\ n}}^{n} \alpha\left(t_{1}, \ldots, t_{r}\right) x_{1}^{t_{1}} \ldots x_{r}^{t_{r}}
$$

получено

$$
\gamma \leqslant r\left(\begin{array}{c}
n+r \\
r+1
\end{array}\right)+r
$$


И. А. Икромовым [8] получены оценки снизу:

$$
\gamma \geqslant \frac{1}{2} n m+1, \quad \gamma \geqslant\left(\begin{array}{l}
n+r \\
r+1
\end{array}\right)+1
$$

для многочленов вида (2) и (3) соответственно. Также в [8] в одном частном случае многочлена $F\left(x_{1}, \ldots, x_{r}\right)$, имеющего вид

$$
F\left(x_{1}, \ldots, x_{r}\right)=\sum_{t_{1}=[n / 2]+1}^{n}\left(\sum_{t_{2}=0}^{t_{1}} \cdots \sum_{t_{r}=0}^{t_{1}} \alpha\left(t_{2}, \ldots, t_{r}\right) x_{2}^{t_{2}} \ldots x_{r}^{t_{r}}\right) x_{1}^{t_{1}},
$$

получено точное значение показателя сходимости $\gamma$ :

$$
\gamma=\sum_{k=[n / 2]+1}^{n} k(k+1)^{r-1}
$$

Пусть $A=\left\{\left(t_{1}, \ldots, t_{r}\right) \mid t_{1} \geqslant 0, \ldots, t_{r} \geqslant 0\right\},(0, \ldots, 0) \notin A,-$ некоторьй конечный набор целочисленных векторов $\left(t_{1}, \ldots, t_{r}\right)$, и пусть

$$
F\left(x_{1}, \ldots, x_{r}\right)=\sum_{\left(t_{1}, \ldots, t_{r}\right) \in A} \alpha\left(t_{1}, \ldots, t_{r}\right) x_{1}^{t_{1}} \ldots x_{r}^{t_{r}} .
$$

Положим $n_{s}=\max \left\{t_{s} \mid\left(t_{1}, \ldots, t_{s}\right) \in A\right\}, s=1, \ldots, r$.

В п. 3 следуюшие теоремы 1,2 дают оценку снизу показателя сходимости $\gamma$ особого интеграла $\theta$ для неполного и полного многочлена $F\left(x_{1}, \ldots, x_{r}\right)$.

Теорема 1. Пусть интеграл (1) для функиии (7) сходится, и пусть вектор $\left(0, \ldots, 0, n_{s}, 0, \ldots, 0\right)$ принадлежит множеству $A$. Тогда

$$
2 k \geqslant \sum_{\left(t_{1}, \ldots, t_{r}\right) \in A} t_{s}
$$

ТЕОрема 2. Пусть выполнены условия теоремы 1 , и пусть множество $A$ таково, что если вектор $\left(t_{1}, \ldots, n, \ldots, t_{r}\right)$ принадлежст множеству $A$, то векторы $\left(t_{1}, \ldots, t_{s}, \ldots, t_{r}\right)$ тоже принадлежат $A$ при всех $t_{s}, 0 \leqslant t_{s} \leqslant n$. Тогда

$$
2 k \geqslant \sum_{\left(t_{1}, \ldots, t_{r}\right) \in A} t_{s}+1 .
$$

Нетрудно видеть, что для многочленов вида (2) и (3) из теоремы 2 следуют оценки (4). Для неполного многочлена (5) теорема 1 дает точную оценку снизу (6) показателя сходимости особого интеграла. Вместе с тем, теоремы 1, 2 применимы для многочленов $F\left(x_{1}, \ldots, x_{r}\right)$ более обшего вида.

В п. 4 получена оценка сверху показателя сходимости $\gamma$ особого интеграла (1) при более сильных, чем в теореме 1 , ограничениях на множество $A$ (теорема 3 ). Тем не менее, имеется достаточно широкий класс функций, для которых эти требования выполнены. В этих случаях оценки снизу и сверху совпадают, что дает точное значение показателя сходимости $\gamma$ (см. п. 5, теорема 4). 
2. Оценки интегралов от осциллирующих функций. Рассмотрим интеграл

$$
I=\int_{a}^{b} g(x) \exp \{2 \pi i f(x)\} d x
$$

где $f(x)$ - вещественная функция, $a$ - конечное число, $b$ - конечное число или $b=+\infty$.

Существуют различные методы оценки интегралов такого вида (метод перевала, метод стационарной фазы Лапласа; см. [9]), однако они предполагают наличие достаточного числа производных или даже аналитичность функций $f(x)$ и $g(x)$. Между тем, требование только выпуклости функций $f(x)$ позволяет получать оценки интеграла (8), применимые даже для функций $g(x)$ с неограниченной вариацией.

ЛЕмма 1. Пусть функция $f(x)$ монотонно возрастает и выпукла вниз на промежутке $[a, b)$, и пусть $a<x_{1}<x_{2}<\cdots<x_{k}<\cdots<b$ u $a<t_{1}<$ $t_{2}<\cdots<t_{s}<\cdots<b-$ последовательные корни уравнений $\sin (2 \pi f(x))=0 u$ $\cos (2 \pi f(t))=0$ соответственно. (При $f(b-0)<+\infty$ корней конечное число или их нет вовсе.) Тогда найдутся точки $\xi_{k} \in\left(x_{k}, x_{k+1}\right)$ и $\tau_{s} \in\left(t_{s}, t_{s+1}\right)$ такие, что

$$
\int_{\xi_{k}}^{\xi_{k+1}} \sin (2 \pi f(x)) d x=0, \quad \int_{\tau_{s}}^{\tau_{s+1}} \cos (2 \pi f(t)) d t=0, \quad k, s=1,2,3, \ldots
$$

Эта лемма позволяет в действительной и мнимой частях интеграла (8) аппроксимировать функцию $g(x)$ произвольной кусочно постоянной на интервалах $\left(\tau_{s}, \tau_{s+1}\right)$ и $\left(\xi_{k}, \xi_{k+1}\right)$ соответственно функцией.

ДокАЗАТЕЛЬСТво. І. Без ограничения общности будем считать, что $a=0$, $f(0)=0$, и пусть сначала

$$
f(b-0)=\lim _{\substack{x \rightarrow b \\ x<b}} f(x)=+\infty .
$$

Докажем первое соотношение в (9). Второе доказывается аналогично. Рассмотрим функцию

$$
\varphi(x)=\int_{0}^{x} \sin (2 \pi f(t)) d t .
$$

Функция $\varphi(x)$ возрастает на интервалах $\left(x_{k}, x_{k+1}\right)$ при четном $k$ и убывает при нечетном $k$, т.е. точки $x_{k}$ - локальные максимумы функции $\varphi(x)$ при нечетном $k$ и минимумы при четном $k$. Кроме того, максимумы функций $\varphi(x)$ образуют монотонно убывающую, а минимумы - монотонно возрастающую последовательности. Действительно, пусть сначала функция $f(x)$ имеет монотонно возрастающую производную $f^{\prime}(x)$. Тогда

$$
\begin{aligned}
\varphi\left(x_{k+2}\right)-\varphi\left(x_{k}\right) & =\int_{x_{k}}^{x_{k+1}} \sin (2 \pi f(x)) d x+\int_{x_{k+1}}^{x_{k+2}} \sin (2 \pi f(x)) d x \\
& =\int_{k / 2}^{(k+1) / 2} \frac{\sin (2 \pi t)}{f^{\prime}(x(t))} d t+\int_{(k+1) / 2}^{(k+2) / 2} \frac{\sin (2 \pi t) d t}{f^{\prime}(x(t))} \\
& =(-1)^{k} \int_{0}^{1 / 2} \sin (2 \pi t)\left(\frac{1}{f^{\prime}\left(x\left(t+\frac{k}{2}\right)\right)}-\frac{1}{f^{\prime}\left(x\left(t+\frac{k+1}{2}\right)\right)}\right) d t
\end{aligned}
$$


и, следовательно, $\varphi\left(x_{k+2}\right)-\varphi\left(x_{k}\right) \geqslant 0$ при четном $k$ и $\varphi\left(x_{k+2}\right)-\varphi\left(x_{k}\right) \leqslant 0$ при нечетном $k$. В обшем случае достаточно рассмотреть средние Стеклова

$$
f_{h}(x)=\frac{1}{2 h} \int_{-h}^{h} f(x+t) d t
$$

функции $f(x)$, сохраняющие монотонность и выпуклость.

Пусть $c_{1}=\lim _{k \rightarrow \infty} \varphi\left(x_{2 k}\right) \leqslant \lim _{k \rightarrow \infty} \varphi\left(x_{2 k+1}\right)=c_{2}$.

Рассмотрим функцию

$$
g(x)=\varphi(x)-c=\int_{0}^{x} \sin (2 \pi f(t)) d t-c,
$$

где $c \in\left(c_{1}, c_{2}\right)$ при $c_{1} \neq c_{2}$ и $c=c_{1}$ при $c_{1}=c_{2}$. Тогда $g\left(x_{2 s-1}\right)>0$ и $g\left(x_{2 s}\right)<0$, $s=1,2,3, \ldots$, и, следовательно, на каждом интервале $\left(x_{k}, x_{k+1}\right)$ найдется точка $\xi_{k}$ такая, что

$$
\int_{0}^{\xi_{k}} \sin (2 \pi f(t)) d t=c
$$

и, значит,

$$
\int_{\xi_{k}}^{\xi_{k+1}} \sin (2 \pi f(t)) d t=0, \quad k=1,2,3, \ldots
$$

II. В случае $f(b-0)<+\infty$ (ясно, что тогда $b<+\infty$, так как $f(x)$ монотонно возрастает и выпукла вниз) положим

$$
c=\int_{0}^{b} \sin (2 \pi f(t)) d t
$$

Далее доказательство аналогично доказательству, проведенному в п. І. Лемма доказана.

ЗАМЕЧАнИЕ 1. Если функция $f(x)$ монотонна (возрастает или убывает) и выпукла (вверх или вниз) на конечном отрезке $[a, b]$, то лемма 1 остается справедливой, поскольку функция $\pm f( \pm x)$ удовлетворяет условиям леммы 1 при соответствуюшем выборе знаков.

В качестве иллюстрации применения леммы 1 рассмотрим интеграл

$$
I(\lambda)=\int_{0}^{1} g(x) \exp \left\{2 \pi i \lambda x^{\beta}\right\} d x, \quad \lambda \rightarrow \infty
$$

где $\beta>1$ - вещественное число, $g(x) \in \operatorname{Lip}_{\alpha}[0,1], 0<\alpha \leqslant 1$. Определим точки $x_{k}=(k / 2 \lambda)^{1 / \beta}, k=1,2,3, \ldots,[2 \lambda]$, и пусть

$$
c_{\beta}=\int_{0}^{+\infty} \exp \left\{2 \pi i x^{\beta}\right\} d x
$$


По лемме 1 получим

$$
\operatorname{Im} I(\lambda)=\frac{g(0) \operatorname{Im} c_{\beta}}{\lambda^{1 / \beta}}+\sum_{k=0}^{[2 \lambda]-1} \int_{\xi_{k}}^{\xi_{k+1}}\left(g(x)-g\left(\xi_{k}\right)\right) \sin \left(2 \pi \lambda x^{\beta}\right) d x,
$$

где $\xi_{0}=0, \xi_{k} \in\left(x_{k}, x_{k+1}\right), k=1,2,3, \ldots,[2 \lambda]-1, \xi_{[2 \lambda]}=1$. Учитывая, что $\Delta \xi_{k} \leqslant 2 \Delta x_{k}, g(x) \in \operatorname{Lip}_{\alpha}[0,1]$, получим

$$
\operatorname{Im} I(\lambda)=\frac{g(0)}{\lambda^{1 / \beta}} \operatorname{Im} c_{\beta}+O\left(\sum_{k=1}^{[2 \lambda]-1} \frac{1}{\left(k^{\beta-1} \lambda\right)^{(1+\alpha) / \beta}}\right) .
$$

Вычисляя сумму и получая аналогичную оценку для $\operatorname{Re} I(\lambda)$, находим

$$
I(\lambda)=\frac{c_{\beta} g(0)}{\lambda^{1 / \beta}}+O(R), \quad \lambda \rightarrow \infty,
$$

где $R=1 / \lambda^{\alpha}$ при $1<\beta<2,0<\alpha \leqslant 1$ и при $\beta \geqslant 2,0<\alpha<(\beta-1)^{-1}$; $R=(\ln \lambda) / \lambda^{\alpha}$ при $\beta \geqslant 2, \alpha=(\beta-1)^{-1} ; R=1 / \lambda^{(1+\alpha) / \beta}$ при $\beta>2, \quad(\beta-$ $1)^{-1}<\alpha \leqslant 1$.

Рассмотрим тригонометрический интеграл

$$
I=I\left(\alpha_{1}, \ldots, \alpha_{n}\right)=\int_{0}^{1} \exp \{2 \pi i f(x)\} d x
$$

где $f(x)=\alpha_{n} x^{n}+\alpha_{n-1} x^{n-1}+\cdots+\alpha_{1} x-$ многочлен степени $n$ с вещественными коэффициентами.

ЛЕмма 2. При $\left|\alpha_{n}\right|>1$ справедливо представление

$$
\begin{aligned}
& I\left(\alpha_{1}, \ldots, \alpha_{n}\right)=\frac{c_{n}}{\left|\alpha_{n}\right|^{1 / n}}+ \\
& +O\left(\frac{\left|\alpha_{1}\right|}{\left|\alpha_{n}\right|^{2 / n}}+\cdots+\frac{\left|\alpha_{n-2}\right|}{\left|\alpha_{n}\right|^{(n-1) / n}}+\frac{\left|\alpha_{n-1}\right|}{\left|\alpha_{n}\right|} \ln \left|\alpha_{n}\right|+\frac{1}{\left|\alpha_{n}\right|}\right), \\
& c_{n}=\int_{0}^{+\infty} \exp \left\{2 \pi i x^{n} \operatorname{sign} \alpha_{n}\right\} d x .
\end{aligned}
$$

ДокАЗАТЕЛЬСТво. Пусть для определенности $\alpha_{n}>1$. Представим интеграл из (10) в следуюшем виде:

$$
I=\int_{0}^{1} G(x) \cos \left(2 \pi \alpha_{n} x^{n}\right) d x+i \int_{0}^{1} G(x) \sin \left(2 \pi \alpha_{n} x^{n}\right) d x
$$

где $G(x)=\exp \left\{2 \pi i\left(\alpha_{n-1} x^{n-1}+\cdots+\alpha_{1} x\right)\right\}$. Рассмотрим интеграл

$$
I_{1}=\int_{0}^{1} G(x) \sin \left(2 \pi \alpha_{n} x^{n}\right) d x
$$


По лемме 1 имеем

$$
I_{1}=G(0) \int_{0}^{\xi_{1}} \sin \left(2 \pi \alpha_{n} x^{n}\right) d x+\sum_{k=0}^{m} \int_{\xi_{k}}^{\xi_{k+1}}\left[G(x)-G\left(\xi_{k}\right)\right] \sin \left(2 \pi \alpha_{n} x^{n}\right) d x
$$

где $\xi_{0}=0, \xi_{m+1}=1, m=\left[2 \alpha_{n}\right], \xi_{k} \in\left(x_{k}, x_{k+1}\right), x_{k}=\left(\frac{k}{2 \alpha_{n}}\right)^{1 / n}, k=$ $1,2,3, \ldots, m-1$. Поскольку $G(0)=1$ и

$$
\int_{0}^{\xi_{1}} \sin \left(2 \pi \alpha_{n} x^{n}\right) d x=\int_{0}^{1} \sin \left(2 \pi \alpha_{n} x^{n}\right) d x
$$

получаем

$$
I_{1}=\int_{0}^{1} \sin \left(2 \pi \alpha_{n} x^{n}\right) d x+O(R)
$$

где

$$
R=\sum_{k=0}^{m} \int_{\xi_{k}}^{\xi_{k+1}}\left|G(x)-G\left(\xi_{k}\right)\right| d x
$$

Применяя к действительной и мнимой частям функции $G(x)$ теорему о среднем и учитывая, что $\Delta \xi_{k} \leqslant 2 \Delta x_{k}$, а $\Delta x_{k} \leqslant x_{k+1} /(k+1)$ в силу выпуклости функции $\alpha_{n} x^{n}$, получаем

$$
R \leqslant C \sum_{k=0}^{m}\left(\left|\alpha_{n-1}\right| x_{k+2}^{n-2}+\cdots+\left|\alpha_{1}\right|\right) \frac{x_{k+1}^{2}}{(k+1)^{2}}
$$

здесь $x_{m+1}=x_{m+2}=1$, а $C$-положительная постоянная, зависяшая только от $n$.

Поскольку $x_{k}=\left(\frac{k}{2 \alpha_{n}}\right)^{1 / n}$, вычисляя сумму, получим

$$
I_{1}=\int_{0}^{1} \sin \left(2 \pi \alpha_{n} x^{n}\right) d x+O\left(\frac{\left|\alpha_{n-1}\right|}{\left|\alpha_{n}\right|} \ln \left|\alpha_{n}\right|+\frac{\left|\alpha_{n-2}\right|}{\left|\alpha_{n}\right|^{(n-1) / n}}+\cdots+\frac{\left|\alpha_{1}\right|}{\left|\alpha_{n}\right|^{2 / n}}\right) .
$$

Аналогичное соотношение выполнено для

$$
I_{2}=\int_{0}^{1} G(x) \cos \left(2 \pi \alpha_{n} x^{n}\right) d x
$$

Учитьвая, что

$$
\int_{0}^{1} \exp \left\{2 \pi i \alpha_{n} x^{n}\right\} d x=\int_{0}^{+\infty} \exp \left\{2 \pi i \alpha_{n} x^{n}\right\} d x+O\left(\frac{1}{\left|\alpha_{n}\right|}\right)
$$

получаем утверждение леммы.

ЗАмЕчАнИЕ 2. Для других коэффищиентов $\alpha_{s}, s=1, \ldots, n-1,\left|\alpha_{s}\right|>1$, выполнено соотношение

$$
\begin{gathered}
\int_{0}^{1} \exp \left\{2 \pi i\left(\alpha_{n} x^{n}+\cdots+\alpha_{1} x\right)\right\} d x=\frac{c_{s}}{\left|\alpha_{s}\right|^{1 / s}}+O\left(\frac{\left|\alpha_{1}\right|}{\left|\alpha_{s}\right|^{2 / s}}+\cdots+\frac{\left|\alpha_{s-1}\right|}{\left|\alpha_{s}\right|^{(s-1) / s}}\right. \\
\left.+\frac{\left|\alpha_{s-1}\right|}{\left|\alpha_{s}\right|} \ln \left|\alpha_{s}\right|+\frac{\left|\alpha_{s+1}\right|}{\left|\alpha_{s}\right|}+\cdots+\frac{\left|\alpha_{n}\right|}{\left|\alpha_{s}\right|}+\frac{1}{\left|\alpha_{s}\right|}\right),
\end{gathered}
$$

где $c_{s}=\int_{0}^{+\infty} \exp \left\{2 \pi i x^{s} \operatorname{sign} \alpha_{s}\right\} d x$. 


\section{3. Оценки снизу показателя сходимости особого интеграла.}

ДокаЗАтЕльСтво теоРемы 1. Рассмотрим интеграл

$$
\theta=\int_{-\infty}^{+\infty} \cdots \int_{-\infty}^{+\infty}\left|\int_{0}^{1} \cdots \int_{0}^{1} \exp \left\{2 \pi i F\left(x_{1}, \ldots, x_{r}\right)\right\} d x_{1} \ldots d x_{r}\right|^{2 k} d \alpha
$$

где

$$
F\left(x_{1}, \ldots, x_{r}\right)=\sum_{\left(t_{1}, \ldots, t_{r}\right) \in A} \alpha\left(t_{1}, \ldots, t_{r}\right) x_{1}^{t_{1}} \ldots x_{r}^{t_{r}} .
$$

Предполагая для определенности, что вектор $(n, 0, \ldots, 0) \in A$, где $n=\max \left\{t_{1} \mid\right.$ $\left.\left(t_{1}, \ldots, t_{r}\right) \in A\right\}$, и положив $x_{1}=x$, запишем многочлен (12) в виде

$$
F\left(x_{1}, x_{2}, \ldots, x_{r}\right)=\sum_{k=0}^{n} \beta_{k} x^{k}
$$

где $\beta_{k}=\sum_{\left(k, t_{2}, \ldots, t_{r}\right) \in A} \alpha\left(k, t_{2}, \ldots, t_{r}\right) x_{2}^{t_{2}} \ldots x_{r}^{t_{r}}$.

Пусть $N=\sum_{\left(t_{1}, \ldots, t_{r}\right) \in A} 1$ - число элементов множества $A$. Запишем интеграл (11) в виде

$$
\theta=\int_{\mathbb{R}^{N}}\left|\int_{0}^{1} \ldots \int_{0}^{1} \exp \left\{2 \pi i \beta_{0}\right\} d x_{2} \ldots d x_{r} \int_{0}^{1} \exp \{2 \pi i f(x)\} d x\right|^{2 k} d \alpha,
$$

где $f(x)=\beta_{n} x^{n}+\cdots+\beta_{1} x$. Определим множество $\Omega \subset \mathbb{R}^{N}$ следующим образом:

$$
\begin{aligned}
\Omega=\{ & \alpha\left(t_{1}, \ldots, t_{r}\right),\left(t_{1}, \ldots, t_{r}\right) \in A \mid \alpha_{n}=\alpha(n, 0, \ldots, 0) \geqslant 2 ; \\
& \left|\alpha\left(n, t_{2}, \ldots, t_{r}\right)\right| \leqslant \frac{\varepsilon}{N} \alpha_{n} ;\left(n, t_{2}, \ldots, t_{r}\right) \neq(n, 0, \ldots, 0) ; \\
& \left|\alpha\left(n-1, t_{2}, \ldots, t_{r}\right)\right| \leqslant \frac{\varepsilon \alpha_{n}^{(n-1) / n}}{N \ln \alpha_{n}} ; \\
& \left.\left|\alpha\left(s, t_{2}, \ldots, t_{r}\right)\right| \leqslant \frac{\varepsilon}{N} \alpha_{n}^{s / n}, s=1, \ldots, n-2 ;\left|\alpha\left(0, t_{2}, \ldots, t_{r}\right)\right| \leqslant \frac{\delta}{N}\right\},
\end{aligned}
$$

где $\varepsilon$ и $\delta$ - положительные числа, которые определим далее. Ясно, что

$$
\theta \geqslant \int_{\Omega}|I(\alpha)|^{2 k} d \alpha
$$

где

$$
I(\alpha)=\int_{0}^{1} \ldots \int_{0}^{1} \exp \left\{2 \pi i \beta_{0}\right\} d x_{2} \ldots d x_{r} \int_{0}^{1} \exp \{2 \pi i f(x)\} d x .
$$

В силу определения множества $\Omega$ нетрудно видеть, что

$$
\begin{gathered}
\left|\beta_{0}\right| \leqslant \delta, \quad\left|\beta_{s}\right| \leqslant \varepsilon \alpha_{n}^{s / n}, \quad s=1, \ldots, n-2, \\
\left|\beta_{n-1}\right| \leqslant \frac{\varepsilon \alpha_{n}^{(n-1) / n}}{\ln \alpha_{n}}, \quad(1-\varepsilon) \alpha_{n} \leqslant \beta_{n} \leqslant(1+\varepsilon) \alpha_{n} .
\end{gathered}
$$


Учитывая, что $\left|1-\exp \left\{2 \pi i \beta_{0}\right\}\right| \leqslant 2 \pi \delta$, для интеграла (13) будем иметь

$$
|I(\alpha)| \geqslant\left|\int_{0}^{1} \exp \{2 \pi i f(x)\} d x\right|-2 \pi \delta\left|\int_{0}^{1} \exp \{2 \pi i f(x)\} d x\right| .
$$

Применяя лемму 2 и учитывая, что

$$
\left|\frac{1}{\beta_{n}^{1 / n}}-\frac{1}{\alpha_{n}^{1 / n}}\right| \leqslant \frac{\varepsilon}{n \alpha_{n}^{1 / n}},
$$

получим

$$
|I(\alpha)| \geqslant \frac{\left|c_{0}\right|}{\alpha_{n}^{1 / n}}+\varepsilon O\left(\frac{1}{\alpha_{n}^{1 / n}}\right)+\delta O\left(\frac{1}{\alpha_{n}^{1 / n}}\right),
$$

где постоянные в знаках $O\left(\alpha_{n}^{-1 / n}\right)$ зависят только от $n$, а

$$
c_{0}=\int_{0}^{+\infty} \exp \left\{2 \pi i x^{n}\right\} d x \neq 0 .
$$

Отсюда получаем, что $|I(\alpha)| \geqslant \frac{\left|c_{0}\right|}{2 \alpha_{n}^{1 / n}}$ при достаточно малых $\varepsilon$ и $\delta$ и всех $\alpha \in \Omega$.

Учитывая определение множества $\Omega$, имеем

$$
\theta \geqslant \frac{\left|c_{0}\right|^{2 k} \varepsilon^{N} \delta^{N}}{2^{2 k} N^{N}} \int_{2}^{+\infty} \frac{1}{\alpha_{n}^{\frac{2 k}{n}-\frac{M-n}{n}} \ln ^{N} \alpha_{n}} d \alpha_{n},
$$

где $M=\sum_{\left(s, t_{2}, \ldots, t_{r}\right) \in A} s$. Поскольку по предположению особый интеграл $\theta$ существует, то для его сходимости необходимо выполнение неравенства $2 k \geqslant M$. Теорема доказана.

ДОКАЗАТЕЛЬСТВО ТЕОРЕМЫ 2. Не ограничивая общности, будем считать, что условия теоремы выполнены при $s=1$, т.е. вектор $(n, 0, \ldots, 0)$ принадлежит множеству $A$, где $n=\max \left\{t_{1} \mid\left(t_{1}, \ldots, t_{r}\right) \in A\right\}$. При фиксированных значениях $t_{2}, \ldots, t_{r}$ положим

$$
m=m\left(t_{2}, \ldots, t_{r}\right)=\max \left\{s \mid\left(s, t_{2}, \ldots, t_{r}\right) \in A\right\},
$$

и пусть $B$ - множество векторов $\left(t_{2}, \ldots, t_{r}\right)$, для которых определено число $m$.

Ясно, что $0 \leqslant m \leqslant n$. В силу условий теоремы функцию

$$
F=F\left(x_{1}, \ldots, x_{r}\right)=\sum_{\left(t_{1}, \ldots, t_{r}\right) \in A} \alpha\left(t_{1}, \ldots, t_{r}\right) x_{1}^{t_{1}} \ldots x_{r}^{t_{r}}
$$

можно представить в виде

$$
F=\sum_{\left(t_{2}, \ldots, t_{r}\right) \in B} x_{2}^{t_{2}} \ldots x_{r}^{t_{r}} \sum_{k=0}^{m\left(t_{2}, \ldots, t_{r}\right)} \alpha\left(k, t_{2}, \ldots, t_{r}\right) x^{k}
$$


где $x=x_{1}$. При целом $P \geqslant 3$ и $s=1,2, \ldots, P$ рассмотрим многочлены

$$
\varphi(x ; s)=\varphi\left(x ; s ; t_{2}, \ldots, t_{r}\right)=\sum_{k=0}^{m} \alpha\left(k, t_{2}, \ldots, t_{r}\right) x^{k}
$$

с коэффициентами $\alpha\left(k, t_{2}, \ldots, t_{r}\right), k=0, \ldots, m$, определяемыми из соотношений

$$
\sum_{k=0}^{m} \alpha\left(k, t_{2}, \ldots, t_{r}\right) x^{k}=\sum_{k=0}^{m} \beta_{k}\left(t_{2}, \ldots, t_{r}\right)\left(x-u_{s}\right)^{k},
$$

где $u_{s}=\frac{1}{4}+\frac{s}{2 P}, s=1, \ldots, P$, а $\beta_{k}=\beta_{k}\left(t_{2}, \ldots, t_{r}\right), k=1,2, \ldots, m, m \leqslant n,-$ любые числа с условиями

$$
\begin{gathered}
\beta_{n}(0, \ldots, 0)=\alpha(n, 0, \ldots, 0)=\alpha_{n} \geqslant P^{n}, \\
0 \leqslant \beta_{n}\left(t_{2}, \ldots, t_{r}\right) \leqslant \frac{\varepsilon}{N} \alpha_{n}, \quad\left(t_{2}, \ldots, t_{r}\right) \neq(0, \ldots, 0), \\
0 \leqslant \beta_{n-1}\left(t_{2}, \ldots, t_{r}\right) \leqslant \frac{\varepsilon}{N} \frac{\alpha_{n}^{(n-1) / n}}{\ln \alpha_{n}}, \\
0 \leqslant \beta_{s}\left(t_{2}, \ldots, t_{r}\right) \leqslant \frac{\varepsilon}{N} \alpha_{n}^{s / n}, \quad s=1,2, \ldots, n-2, \\
0 \leqslant \beta_{0}\left(t_{2}, \ldots, t_{r}\right) \leqslant \frac{\delta}{N}, \quad\left(t_{2}, \ldots, t_{r}\right) \in B
\end{gathered}
$$

где $\varepsilon$ и $\delta$ - достаточно малые положительные числа, которые определим далее.

Обозначим через $\Omega(s), \Omega(s) \subset \mathbb{R}^{N}, s=1,2, \ldots, P$, множество точек $\mathbb{R}^{N}$, координаты которых $\alpha(k)=\alpha\left(k, t_{2}, \ldots, t_{r}\right)$ удовлетворяют неравенствам (15). Отметим, что якобиан перехода от переменных $\alpha(k)$ к переменньг $\beta(k)$ равен 1 . Так же, как в $\left[1\right.$, с. 29], покажем, что при $s_{1} \neq s_{2}$ множества $\Omega\left(s_{1}\right)$ и $\Omega\left(s_{2}\right)$ не пересекаются. Рассмотрим многочлены $\varphi\left(x ; s_{1} ; 0, \ldots, 0\right)$ и $\varphi\left(x ; s_{2} ; 0, \ldots, 0\right)$. Коэффициент многочлена $\varphi(x ; s ; 0, \ldots, 0)$ равен

$$
\alpha(n-1,0, \ldots, 0)=-n\left(\frac{1}{4}+\frac{s}{2 P}\right) \beta_{n}+\beta_{n-1} .
$$

Обозначая через $\alpha_{n-1}^{\prime}$ и $\alpha_{n-1}^{\prime \prime}$ коэффициенты $\alpha(n-1,0, \ldots, 0)$ многочленов $\varphi\left(x ; s_{1}\right)$ и $\varphi\left(x ; s_{2}\right)$ соответственно, будем иметь

$$
\begin{aligned}
\left|\alpha_{n-1}^{\prime}-\alpha_{n-1}^{\prime \prime}\right| & =\left|\frac{n}{2 P}\left(s_{2}-s_{1}\right)+\beta_{n-1}^{\prime}-\beta_{n-1}^{\prime \prime}\right| \geqslant \frac{n}{2 P} \alpha_{n}-\frac{2 \varepsilon}{N} \frac{\alpha_{n}^{(n-1) / n}}{\ln \alpha_{n}} \\
& \geqslant \alpha_{n}^{n-1}\left(\frac{n}{2}-\frac{2 \varepsilon}{N \ln \alpha_{n}}\right)>0
\end{aligned}
$$

и, следовательно, $\Omega\left(s_{1}\right) \cap \Omega\left(s_{2}\right)=\varnothing$. Оценим снизу модуль интеграла

$$
J=\int_{0}^{1} \exp \left\{2 \pi i f\left(x-u_{s}\right)\right\} d x
$$


где

$$
f(x)=\sum_{\left(t_{2}, \ldots, t_{r}\right) \in B} x_{2}^{t_{2}} \ldots x_{r}^{t_{r}} \sum_{k=1}^{m} \beta_{k} x^{k} .
$$

Делая замену переменной, получим

$$
J=\int_{-u_{s}}^{1-u_{s}} \exp \{2 \pi i f(x)\} d x=\int_{0}^{1-u_{s}} \exp \{2 \pi i f(x)\} d x+\int_{0}^{u_{s}} \exp \{2 \pi i f(-x)\} d x .
$$

Запишем многочлен $f(x)$ в виде $f(x)=\gamma_{n} x^{n}+\cdots+\gamma_{1} x$. Поскольку

$$
\gamma_{n}=\sum_{\left(t_{2}, \ldots, t_{r}\right) \in B} \beta_{n}\left(t_{2}, \ldots, t_{r}\right) x_{2}^{t_{2}} \ldots x_{r}^{t_{r}}
$$

а вектор $(n, 0, \ldots, 0) \in A$, то $\beta(n, 0, \ldots, 0) \leqslant \gamma_{n} \leqslant(1+\varepsilon) \beta(n, 0, \ldots, 0)$.

Рассмотрим интеграл

$$
I=\int_{0}^{a} \exp \{2 \pi i f(x)\} d x=a \int_{0}^{1} \exp \{2 \pi i f(a x)\} d x .
$$

Применяя лемму 2 , получим

$$
I=\frac{c_{n}}{\gamma_{n}^{2 / n}}+O\left(\frac{\gamma_{1}}{\gamma_{n}^{2 / n}}+\cdots+\frac{\gamma_{n-2}}{\gamma_{n}^{(n-1) / n}}+\frac{\gamma_{n-1}}{\gamma_{n}} \ln a^{n} \gamma_{n}+\frac{1}{a^{n-2} \gamma_{n}}\right) .
$$

Подставляя $a=1-u_{s}$ и $a=u_{s}$ и учитьвая, что $1 / 4 \leqslant a \leqslant 1$, имеем

$$
J=\frac{c}{\gamma_{n}^{1 / n}}+O\left(\frac{\gamma_{1}}{\gamma_{n}^{2 / n}}+\cdots+\frac{\gamma_{n-2}}{\gamma_{n}^{(n-1) / n}}+\frac{\gamma_{n-1}}{\gamma_{n}} \ln \gamma_{n}+\frac{1}{\gamma_{n}}\right)
$$

где $c=c_{n}+\bar{c}_{n}=2 \int_{0}^{+\infty} \cos \left(2 \pi x^{n}\right) d x$ при нечетном $n$ и $c=2 c_{n}$ при четном $n$. При этом $|c|>\sqrt{2}$ (см. $[1$, с. 31$])$. Заменяя в $(17)$

$$
\gamma_{k}=\sum_{\left(t_{2}, \ldots, t_{r}\right) \in B} x_{2}^{t_{2}} \ldots x_{r}^{t_{r}} \beta_{k}\left(t_{2}, \ldots, t_{r}\right), \quad k=1, \ldots, n
$$

и учитывая ограничения (15), получим

$$
J=\frac{c}{\alpha_{n}^{1 / n}}+\varepsilon O\left(\frac{1}{\alpha_{n}^{1 / n}}\right) .
$$

Кроме того,

$$
\begin{gathered}
\int_{0}^{1} \cdots \int_{0}^{1} J \exp \left\{2 \pi i \beta_{0}\right\} d x_{2} \ldots d x_{r}=\int_{0}^{1} \int_{0}^{1} J d x_{2} \ldots d x_{r} \\
+\int_{0}^{1} \cdots \int_{0}^{1}\left(\exp \left\{2 \pi i \beta_{0}\right\}-1\right) J d x_{2} \ldots d x_{r}= \\
=\frac{c}{\alpha_{n}^{1 / n}}+\varepsilon O\left(\frac{1}{\alpha_{n}^{1 / n}}\right)+\delta O\left(\frac{1}{\alpha_{n}^{1 / n}}\right)
\end{gathered}
$$


поскольку $\beta_{0}=\sum_{\left(t_{2}, \ldots, t_{r}\right)} x_{2}^{t_{2}} \ldots x_{r}^{t_{r}} \beta_{0}\left(t_{2}, \ldots, t_{r}\right) \leqslant \delta$.

Выбирая $\varepsilon$ и $\delta$ достаточно малыми, будем иметь

$$
\left|\int_{0}^{1} \cdots \int_{0}^{1} \exp \left\{2 \pi i F\left(x_{1}, \ldots, x_{r}\right)\right\} d x_{1} \ldots d x_{r}\right| \geqslant \frac{|c|}{2 \alpha_{n}^{1 / n}}
$$

для всех точек множества $\Omega(s)$. Отсюда для особого интеграла $\theta$ получим оценку снизу:

$$
\begin{aligned}
\theta & \geqslant \sum_{s=1}^{p} \int_{\Omega(s)}\left|\int_{0}^{1} \cdots \int_{0}^{1} \exp \left\{2 \pi i F\left(x_{1}, \ldots, x_{r}\right)\right\} d x_{1} \ldots d x_{r}\right|^{2 k} d \alpha \\
& \geqslant \frac{\varepsilon^{N} \delta^{N}}{N^{N}} P \int_{P^{n}}^{+\infty} \frac{d \alpha_{n}}{\alpha_{n}^{(2 k-M+n) / n} \ln ^{N} \alpha_{n}}
\end{aligned}
$$

где $M=\sum_{\left(t_{1}, \ldots, t_{r}\right) \in A} t_{1}$. При $P \rightarrow+\infty$ для сходимости интеграла необходимо, чтобы выполнялось неравенство $2 k \geqslant M+1$. Теорема доказана.

Условия теоремы 2 выполнены для рассмотренных выше классов функций вида (2) и (3), и, следовательно, из теоремы 2 получаем оценки снизу (4) для показателя сходимости особого интеграла. Заметим еще, что полученные оценки снизу показателя сходимости оказались точными во всех случаях, когда известны точные значения показателя сходимости.

4. Оценки сверху для показателя сходимости особого интеграла. В отличие от оценок снизу, оценки сверху удалось получить для многочленов $F\left(x_{1}, \ldots, x_{r}\right)$, или, что то же самое, для множеств $A$ при более жестких ограничениях. Пусть, как и выше,

$$
F\left(x_{1}, \ldots, x_{r}\right)=\sum_{\left(t_{1}, \ldots, t_{r}\right) \in A} \alpha\left(t_{1}, \ldots, t_{r}\right) x_{1}^{t_{1}} \ldots x_{r}^{t_{r}},
$$

и пусть $m_{s}=\sum_{\left(t_{1}, \ldots, t_{r}\right) \in A} t_{s}, n_{s}=\max \left\{t_{s} \mid\left(t_{1}, \ldots, t_{r}\right) \in A\right\}, s=1,2, \ldots, r$.

ТЕОРема 3. Пусть множество А удовлетворяет условиям:

а) если $\left(t_{1}, \ldots, t_{r}\right) \in A$, mo $t_{1} \neq 0$;

б) $е с л и\left(t_{1}, \ldots, t_{r}\right) \neq\left(\tau_{1}, \ldots, \tau_{r}\right)$, mo $t_{1} \neq \tau_{1}$.

Тогда особий интеграл

$$
\theta=\int_{-\infty}^{+\infty} \cdots \int_{-\infty}^{+\infty}\left|\int_{0}^{1} \cdots \int_{0}^{1} \exp \left\{2 \pi i F\left(x_{1}, \ldots, x_{r}\right)\right\} d x_{1} \ldots d x_{r}\right|^{2 k} d \alpha
$$

сходится, если $2 k>\max \left\{m_{1}+1, m_{2}, \ldots, m_{r}\right\}$.

Кроме того, при условии, что

$$
m_{1}<\frac{n_{1}\left(n_{1}+1\right)}{2}
$$

особый интеграл сходится, если $2 k>\max \left\{m_{1}, \ldots, m_{r}\right\}$.

Заметим, что условие (18) означает, что многочлен $F\left(x_{1}, \ldots, x_{r}\right)$ неполный по переменной $x_{1}$. Соответственно, при

$$
m_{1}=\frac{n_{1}\left(n_{1}+1\right)}{2}
$$

многочлен $F\left(x_{1}, \ldots, x_{r}\right)$ является полным, т.е. содержит все степени переменной $x_{1}$ от 1 до $n_{1}$, где $n_{1}=\max \left\{t_{1} \mid\left(t_{1}, \ldots, t_{r}\right) \in A\right\}$. 
ДОКАЗАТЕЛЬСТВО ТЕОРЕМЫ 3. Проведем доказательство индукцией по числу переменных $r$. При $r=1$ теорема в случае неполного (18) и полного (19) многочлена $F(x)$ верна (см. [1, гл. I, теоремы 3 и 4]). Пусть теорема верна для многочлена от $r-1$ переменной $x_{1}, \ldots, x_{r-1}$, т.е. $\theta_{r-1}<+\infty$, и пусть $\Omega(T) \subset \mathbb{R}^{N}$ :

$$
\Omega(T)=\left\{\alpha\left(t_{1}, \ldots, t_{r}\right)|| \alpha\left(t_{1}, \ldots, t_{r}\right) \mid \leqslant T,\left(t_{1}, \ldots, t_{r}\right) \in A\right\},
$$

- $N$-мерный параллелепипед, где $N=\sum_{\left(t_{1}, \ldots, t_{r}\right) \in A} 1$ - число коэффициентов многочлена $F\left(x_{1}, \ldots, x_{r}\right)$. Рассмотрим интеграл

$$
\theta_{r}(T)=\int_{\Omega(T)}\left|\int_{0}^{1} G\left(x_{r}\right) d x_{r}\right|^{2 k} d \alpha
$$

где

$$
G\left(x_{r}\right)=G\left(x_{r}, \alpha\left(t_{1}, \ldots, t_{r}\right)\right)=\int_{0}^{1} \ldots \int_{0}^{1} \exp \left\{2 \pi i F\left(x_{1}, \ldots, x_{r}\right)\right\} d x_{1} \ldots d x_{r-1}
$$

Пусть $0<\varepsilon<1$ - произвольное число. Из неравенства $|a+b|^{2 k} \leqslant 2^{2 k}\left(|a|^{2 k}+\right.$ $\left.|b|^{2 k}\right), k \geqslant 0$, получим

$$
\theta_{r}(T) \leqslant 2^{2 k} \int_{\Omega(T)}\left|\int_{0}^{\varepsilon} G\left(x_{r}\right) d x_{r}\right|^{2 k} d \alpha+2^{2 k} \int_{\Omega(T)}\left|\int_{\varepsilon}^{1} G\left(x_{r}\right) d x_{r}\right|^{2 k} d \alpha .
$$

Рассмотрим первый интеграл. Делая в нем замену переменных $x_{r}=\varepsilon t$, $\alpha\left(t_{1}, \ldots, t_{r}\right)=\varepsilon^{-t_{r}} \beta\left(t_{1}, \ldots, t_{r}\right),\left(t_{1}, \ldots, t_{r}\right) \in A$, получим

$$
I_{1}=\int_{\Omega(T)}\left|\int_{0}^{\varepsilon} G\left(x_{r}\right) d x_{r}\right|^{2 k} d \alpha=\varepsilon^{2 k-m_{r}} \int_{\Omega\left(\varepsilon^{\left.t_{r} T\right)}\right.}\left|\int_{0}^{1} G(t) d t\right|^{2 k} d \beta .
$$

Поскольку $\Omega\left(\varepsilon^{t_{r}} T\right) \subset \Omega(T)$, то

$$
I_{1} \leqslant \varepsilon^{2 k-m_{r}} \int_{\Omega(T)}\left|\int_{0}^{1} G(t) d t\right|^{2 k} d \beta=\varepsilon^{2 k-m_{r}} \theta_{r}(T) .
$$

Рассмотрим второй интеграл из формулы (20). Применяя ко внутреннему интегралу неравенство Гёльдера и меняя порядок интегрирования, получим

$$
\begin{aligned}
I_{2} & =\int_{\Omega(T)}\left|\int_{\varepsilon}^{1} G\left(x_{r}\right) d x_{r}\right|^{2 k} d \alpha \leqslant(1-\varepsilon)^{2 k-1} \int_{\Omega(T)}\left(\int_{\varepsilon}^{1}\left|G\left(x_{r}\right)\right|^{2 k} d x_{r}\right) d \alpha \\
& \leqslant(1-\varepsilon)^{2 k-1} \int_{\varepsilon}^{1}\left(\int_{\Omega(T)}\left|G\left(x_{r}\right)\right|^{2 k} d \alpha\right) d x_{r} .
\end{aligned}
$$


Во внутреннем интеграле сделаем замену $\alpha\left(t_{1}, \ldots, t_{r}\right) x_{r}^{t_{r}}=\beta\left(t_{1}, \ldots, t_{r}\right)$, $\left(t_{1}, \ldots, t_{r}\right) \in A$. Учитывая, что

$$
\begin{aligned}
& G\left(x_{r}\right)=G\left(x_{r}, \alpha\right)= \\
& \quad=\int_{0}^{1} \ldots \int_{0}^{1} \exp \left\{2 \pi i \sum_{\left(t_{1}, \ldots, t_{r}\right) \in A} \alpha\left(t_{1}, \ldots, t_{r}\right) x_{1}^{t_{1}} \ldots x_{r}^{t_{r}}\right\} d x_{1} \ldots d x_{r-1} \\
& \quad=\int_{0}^{1} \ldots \int_{0}^{1} \exp \left\{2 \pi i \sum_{\left(t_{1}, \ldots, t_{r}\right) \in A} \beta\left(t_{1}, \ldots, t_{r}\right) x_{1}^{t_{1}} \ldots x_{r-1}^{t_{r-1}}\right\} d x_{1} \ldots d x_{r-1}=G(1, \beta)
\end{aligned}
$$

получим

$$
I_{2} \leqslant(1-\varepsilon)^{2 k-1} \int_{\varepsilon}^{1} x_{r}^{-m_{r}}\left(\int_{\Omega\left(T_{1}\right)}|G(1, \beta)|^{2 k} d \beta\right) d x_{r}
$$

где $\Omega\left(T_{1}\right)=\left\{\beta\left(t_{1}, \ldots, t_{r}\right)|| \beta\left(t_{1}, \ldots, t_{r}\right) \mid \leqslant x_{r}^{t_{r}} T,\left(t_{1}, \ldots, t_{r}\right) \in A\right\}$.

Нетрудно видеть, что многочлен от $r-1$ переменной

$$
F\left(x_{1}, \ldots, x_{r-1}, 1\right)=\sum_{\left(t_{1}, \ldots, t_{r}\right) \in A} \beta\left(t_{1}, \ldots, t_{r}\right) x_{1}^{t_{1}} \ldots x_{r-1}^{t_{r-1}}
$$

удовлетворяет всем требованиям теоремы. По предположению индукции отсюда имеем

$$
\begin{aligned}
\int_{\Omega\left(T_{1}\right)} & |G(1, \beta)|^{2 k} d \beta \leqslant \\
& \leqslant \int_{\mathbb{R}^{N}}\left|\int_{0}^{1} \cdots \int_{0}^{1} \exp \left\{2 \pi i F\left(x_{1}, \ldots, x_{r-1}, 1\right)\right\} d x_{1} \ldots d x_{r-1}\right|^{2 k} d \beta=\theta_{r-1} .
\end{aligned}
$$

Особый интеграл $\theta_{r-1}$ сходится при $2 k>\max \left\{m_{1}, \ldots, m_{r-1}\right\}$ в случае (18) и при $2 k>\max \left\{m_{1}+1, m_{2}, \ldots, m_{r-1}\right\}$ в случае выполнения равенства (19). Отсюда

$$
I_{2} \leqslant(1-\varepsilon)^{2 k-1} \theta_{r-1} \int_{\varepsilon}^{1} x_{r}^{-m_{r}} d x_{r} \leqslant \frac{(1-\varepsilon)^{2 k-1}}{\left(m_{r}-1\right) \varepsilon^{m_{r}-1}} \theta_{r-1} .
$$

Подставляя соотношения (21) и (22) в (20), получим

$$
\theta_{r}(T) \leqslant 2^{2 k} \varepsilon^{2 k-m_{r}} \theta_{r}(T)+\frac{2^{2 k}(1-\varepsilon)^{2 k-1}}{\left(m_{r}-1\right) \varepsilon^{m_{r}-1}} \theta_{r-1} .
$$

Поскольку по условию $2 k-m_{r}>0$, то можно выбрать $\varepsilon, 0<\varepsilon<1$, столь малым, что будет выполняться неравенство $2^{2 k} \varepsilon^{2 k-m_{r}}<1$. Тогда

$$
\theta_{r}(T) \leqslant \frac{2^{2 k}(1-\varepsilon)^{2 k-1}}{\left(m_{r}-1\right) \varepsilon^{m_{r}-1}\left(1-2^{2 k} \varepsilon^{2 k-m_{r}}\right)} \theta_{r-1} .
$$

Отсюда $\theta_{r}=\lim _{T \rightarrow+\infty} \theta_{r}(T)<+\infty$. Теорема доказана. 
5. Точные значения показателя сходимости. Из теорем 1 и 3 непосредственно следует

Теорема 4. Пусть множество А челочисленных векторов $\left(t_{1}, \ldots, t_{r}\right)$ удовлетворяет условиям:

1) $(n, 0, \ldots, 0) \in A$, где $n=\max \left\{t_{1} \mid\left(t_{1}, \ldots, t_{r}\right) \in A\right\}$;

2) если $\left(t_{1}, \ldots, t_{r}\right) \in A$, mo $t_{1} \neq 0$;

3) если $\left(t_{1}, \ldots, t_{r}\right) \neq\left(\tau_{1}, \ldots, \tau_{r}\right)$, mo $t_{1} \neq \tau_{1}$;

4) $\max \left\{m_{1}, m_{2}, \ldots, m_{r}\right\}=m_{1}<\frac{n(n+1)}{2}$, где $m_{s}=\sum_{\left(t_{1}, \ldots, t_{r}\right) \in A} t_{s}$.

Тогда для показателя сходимости особого интеграла $\theta$ функиии

$$
F\left(x_{1}, \ldots, x_{r}\right)=\sum_{\left(t_{1}, \ldots, t_{r}\right) \in A} \alpha\left(t_{1}, \ldots, t_{r}\right) x_{1}^{t_{1}} \ldots x_{r}^{t_{r}}
$$

имеем $\gamma=m_{1}$.

Существует достаточно большой класс функций, удовлетворяюших условиям теоремы 4. Однако функции вида (2) и (3) в него не входят. Тем самым вопрос о точном значении показателя сходимости для них остается открытым

Автор благодарен А. А. Карацубе за полезные обсуждения и М.-С. Плиеву за постоянное стимулирование к оформлению настоящей работы.

\section{Список литературы}

1. Архипов Г.И., Карацуба А. А., Чубариков В. Н. Теория кратных тригонометрических сумм. М.: Наука, 1987.

2. Архипов Г.И., Карачуба А.А., Чубариков В.Н. Показатель сходимости особого интеграла проблемы Терри // ДАН СССР. 1979. Т. 248. № 2. С. 268-272.

3. Архипов Г. И., Карачуба А. А., Чубариков В. Н. Кратные тригонометрические суммы. М.: Наука, 1980.

4. Архипов Г.И., Карачуба А.А., Чубариков В.Н. Тригонометрические интегралы // Изв. АН СССР. Сер. матем. 1979. Т. 43. № 5. С. 971-1003.

5. Чубариков В. Н. Об одном кратном тригонометрическом интеграле // ДАН СССР. 1976. T. 227. №6. C. 1308-1310.

6. Чубариков B. H. О кратных рациональных тригонометрических суммах и кратных интегралах // Матем. заметки. 1976. Т. 20. № 1. С. 61-68.

7. Hua Loo-Keng. On the numbers of solutions of Tarry's problem // Acta Sci. Sinica. 1952. V. 1. № 1. P. 1-76.

8. Икромов И. А. On the convergence exponent of trigonometric integrals // Тр. МИАН. 1997. T. 218. C. $179-189$.

9. Эрдейи А. Асимптотические разложения. М.: Наука, 1962.

Московский государственный социальный университет

Поступило в редакцию 2.VII.2002 